\title{
Red blood cell abnormalities and hypertension
}

\author{
Kazushi Tsuda ${ }^{1,2}$
}

Received: 18 September 2019 / Revised: 25 September 2019 / Accepted: 27 September 2019 / Published online: 30 October 2019

(c) The Japanese Society of Hypertension 2019

It is well recognized that abnormalities in the functional and physicochemical properties of red blood cells (RBCs) may underlie the defects that are strongly linked to hypertension, stroke, and other cardiovascular diseases. Odashiro et al. [1] showed that the deformability of circulating RBCs might be impaired in nondiabetic hypertensive patients by a nickel mesh filtration technique [1]. Regarding membrane transport systems, alterations in sodium-lithium countertransport, sodium-potassium ATPase activity, and other electrolyte exchanges through RBC membranes have been demonstrated in hypertension [2-5]. Radosinska and Vrbjar showed that RBC deformability might be closely related to the sodium-potassium ATPase activity of RBC membranes [3]. Junqueira et al. [6] observed that by using the technique of nailfold videocapillaroscopy, the RBC velocity value might be lower in patients with resistant hypertension than in those with mild-to-moderate hypertension and normotensive subjects. It is strongly suggested that functional abnormalities in RBCs could, at least in part, contribute to microcirculatory dysfunction in hypertension.

On the other hand, our studies with an electron spin resonance method have shown that the membrane fluidity of RBCs might be reduced in subjects with essential hypertension compared with normotensive subjects [7-11]. Membrane fluidity (a reciprocal value of membrane microviscosity) is a physicochemical feature of biomembranes that is an important factor in modulating cell rheologic behavior. Changes in membrane fluidity and microviscosity might also affect membrane permeability, transport systems, receptor functions, or enzyme activities [12-14]. There are suggestions that

Kazushi Tsuda

tsudak@mail.wakayama-med.ac.jp

1 Cardiovascular Medicine, Cardiovascular and Metabolic Research Center, Kansai University of Health Sciences, Osaka 590-0482, Japan

2 Division of Cardiology, School of Medicine, Wakayama Medical University, Wakayama 641-8509, Japan membrane fluidity can be modified by various factors, such as membrane components, cytoskeletal proteins, intracellular calcium and sodium contents, or a variety of neurohumoral molecules [12-14]. In a study presented previously, we showed that the membrane fluidity of RBCs as well as that of vascular smooth muscle cells was also lower in spontaneously hypertensive rats than in normotensive Wistar-Kyoto rats at both young and adult ages [15]. It might be possible that cell membranes might be stiffer and less fluid in primary hypertension. The reduced membrane fluidity of RBCs was not observed in subjects with secondary hypertension [16], suggesting that the abnormalities may not be a consequence of a state of high blood pressure. Because the deformability of RBCs might be highly dependent on membrane fluidity, the reduction in membrane fluidity of RBCs could cause a disturbance in blood rheologic behavior and in oxygen perfusion in microcirculation, which would contribute to the pathophysiology of hypertension [12-14].

Recently, it has been shown that red blood cell distribution width (RDW), a measure of variation of RBC size, might be a predictor of cardiovascular and cerebrovascular diseases $[17,18]$. Previous studies have already indicated a strong correlation between higher RDW and higher levels of blood pressure [18, 19]. However, few longitudinal studies have been conducted on the relationship between RDW and incidence of hypertension. A study by Seo et al. in this issue of Hypertension Research [20] demonstrated that high RDW might be significantly and independently associated with the development of hypertension in Korean adults. They analyzed 124,261 participants without hypertension at baseline who underwent two health checkups at 4-year intervals. The mean age of the participants was $37.0 \pm 6.3$ years, and the proportion of males was $55.9 \%$. The means of systolic and diastolic blood pressure were $108 \pm 11$ and $69 \pm 8 \mathrm{mmHg}$, respectively. First, the authors demonstrated that systolic and diastolic blood pressure changes and the numbers of new patients on hypertension medication after 4 years increased as the levels of baseline RDW increased. In multivariate logistic regression analysis, the odds ratio for incident hypertension was 1.15 per $1 \%$ increase in RDW. 
Second, the association between RDW and incident hypertension was similar across clinically relevant subgroups. There were no significant interactions according to age, sex, body mass index, diabetes, current smoking habit, alcohol intake, vigorous exercise two or more times per week, or high-sensitive C-reactive protein value.

The underlying mechanisms responsible for the association between high RDW and hypertension are uncertain. There has been much evidence showing that nitric oxide $(\mathrm{NO})$ might actively participate in the regulation of RBC physiology $[8,10,21]$. It was demonstrated that the RBCs of humans might be positive for NO synthase [21], suggesting that RBCs could synthesize NO and modulate microvascular function. We reported that the lower membrane fluidity of RBCs was associated with decreased levels of plasma NO metabolites [8]. In an in vitro study, it was shown that exogenously applied NO donors significantly increased the membrane fluidity of RBCs in hypertensive subjects [10]. The findings might support the hypothesis that $\mathrm{NO}$ would be a determinant of RBC functions, although the precise relationships between high RDW and endothelial function or NO bioavailability are to be clarified.

In summary, Seo et al. clearly demonstrated that high RDW was associated with an increased risk of incident hypertension in a large retrospective cohort study. The association was independent of established risk factors and was progressive with increased RDW. Although we have a very limited understanding of the mechanisms accounting for the relationship between RBC abnormalities and hypertension, a better knowledge of the cellular mechanisms underlying RBC abnormalities could provide useful information concerning the development of a more specific and more physiological approach to hypertension research.

\section{Compliance with ethical standards}

Conflict of interest The author declares that he has no conflict of interest.

Publisher's note Springer Nature remains neutral with regard to jurisdictional claims in published maps and institutional affiliations.

\section{References}

1. Odashiro K, Saito K, Arita T, Maruyama T, Fujino T, Akashi K. Impaired deformability of circulating erythrocytes obtained from nondiabetic hypertensive patients: investigation by a nickel mesh filtration technique. Clin Hypertens. 2015;21:17.

2. Semplicini A, Sartori M, Ceolotto G, Calò LA. The $\mathrm{Li}^{+} / \mathrm{Na}^{+}$ exchange in hypertension. Front Biosci. 2003;8:d912-29.

3. Radosinska J, Vrbjar N. The role of red blood cell deformability and $\mathrm{Na}, \mathrm{K}$-ATPase function in selected risk factors of cardiovascular diseases in humans: focus on hypertension, diabetes mellitus and hypercholesterolemia. Physiol Res. 2016;65 (Suppl 1):S43-54.

4. Günther T. Mechanisms, regulation and pathological significance of $\mathrm{Mg}^{2+}$ efflux from erythrocytes. Magnes Res. 2006;19:190-8.

5. Zheng X, Chen X, Zhao C, Zhu D, Zhang J, Zhuo JL. Genetic and genomic evidence for an important role of the $\mathrm{Na}^{+} / \mathrm{H}^{+}$exchanger 3 in blood pressure regulation and angiotensin II-induced hypertension. Physiol Genomics. 2019;51:97-108.

6. Junqueira CLC, Magalhães MEC, Brandão AA, Ferreira E, Cyrino FZGA, Maranhão PA, et al. Microcirculation and biomarkers in patients with resistant or mild-to moderate hypertension: a crosssectional study. Hypertens Res. 2018;41:515-23.

7. Tsuda K, Iwahashi H, Minatogawa $Y$, Nishio I, Kido R, Masuyama Y. Electron spin resonance studies of erythrocytes from spontaneously hypertensive rats and humans with essential hypertension. Hypertension. 1987;9(Suppl III):III19-24.

8. Tsuda K, Kimura K, Nishio I, Masuyama Y. Nitric oxide improves membrane fluidity of erythrocytes in essential hypertension-an electron paramagnetic resonance investigation. Biochem Biophys Res Commun. 2000;275:946-54.

9. Tsuda K, Kinoshita Y, Kimura K, Nishio I, Masuyama Y. Electron paramagnetic resonance investigation on modulatory effect of $17 \beta$ estradiol on membrane fluidity of erythrocytes in postmenopausal women. Arterioscler Thromb Vasc Biol. 2001;21:1306-12.

10. Tsuda K, Nishio I. An association between plasma asymmetric dimethylarginine and membrane fluidity of erythrocytes in hypertensive and normotensive men. Am J Hypertens. 2005; 18:1243-8.

11. Tsuda K. Association of resistin with impaired membrane fluidity of red blood cells in hypertensive and normotensive subjects: an electron paramagnetic resonance study. Heart Vessels. 2016;31:1724-30.

12. Zicha J, Kunes J, Devynck MA. Abnormalities of membrane function and lipid metabolism in hypertension. Am J Hypertens. 1999;12:315-31.

13. Tsuda K, Nishio I. Membrane fluidity and hypertension. Am J Hypertens. 2003;16:259-61.

14. Luneva OG, Brazhe NA, Maksimova NV, Rodnenkov OV, Parshina EY, Bryzgalova NY, et al. Ion transport, membrane fluidity and haemoglobin conformation in erythrocyte from patients with cardiovascular diseases: role of augmented plasma cholesterol. Pathophysiology. 2007;14:41-6.

15. Tsuda K, Tsuda S, Minatogawa Y, Iwahashi H, Kido R, Masuyama Y. Decreased membrane fluidity of erythrocytes and cultured vascular smooth muscle cells in spontaneously hypertensive rats: an electron spin resonance study. Clin Sci. 1988;75:477-80.

16. Tsuda K, Tsuda S, Minatogawa Y, Iwahashi H, Shima H, Yoshikawa $\mathrm{H}$, et al. Membrane fluidity of erythrocytes and its relevance to renin profile in essential hypertension. Jpn Circ J. 1988;52:1301-8.

17. Li N, Zhou H, Tang Q. Red blood cell distribution width: a novel predictive indicator for cardiovascular and cerebrovascular diseases. Dis Markers. 2017;2017:7089493.

18. Danese E, Lippi G, Montagnana M. Red blood cell distribution width and cardiovascular diseases. Thorac Dis. 2015;7:E402-11.

19. Gunebakmaz O, Kaya MG, Duran M, Akpek M, Elcik D, Eryol NK. Red blood cell distribution width in 'non-dippers' versus 'dippers'. Cardiology. 2012;123:154-9.

20. Seo SG, Lee MY, Park SH, Han JM, Lee KB, Kim H, et al. The association between red cell distribution width and incident hypertension. Hypertens Res. 2019. https://doi.org/10.1038/ s41440-019-0334-3 [Epub ahead of print].

21. Kleinbongard P, Schulz R, Rassaf T, Lauer T, Dejam A, Jax T, et al. Red blood cells express a functional endothelial nitric oxide synthase. Blood. 2006;107:2943-51. 